\title{
A Note on the Height of Transitive Depth-One Graded Lie Algebras Generated by Their Local Parts
}

\author{
Thomas B. Gregory \\ Department of Mathematics, The Ohio State University at Mansfield, Mansfield, USA \\ Email: gregory.6@osu.edu \\ Received 29 March 2014; revised 28 April 2014; accepted 6 May 2014 \\ Copyright (C) 2014 by author and Scientific Research Publishing Inc. \\ This work is licensed under the Creative Commons Attribution International License (CC BY). \\ http://creativecommons.org/licenses/by/4.0/ \\ c) (i) Open Access
}

\section{Abstract \\ For a transitive depth-one graded Lie algebra over a field of characteristic greater than two, a limit on the degree of the highest gradation space is determined.}

\section{Keywords}

\section{Graded Lie Algebras}

Let the characteristic $p$ of the base field be greater than two. In [1], V.G. Kac defined the local part of a graded Lie algebra $L=L_{-1}+L_{0}+L_{1}+\cdots+L_{k}$ to be $L_{-1}+L_{0}+L_{1}$, which we will assume to be finite dimensional. We will refer to $k$ as the height of $L$.

Lemma 1. If $L=L_{-1}+L_{0}+L_{1}+\cdots+L_{k}$ is a transitive graded Lie algebra which is generated by its finitedimensional local part, then the height $k$ of $L$ is less than or equal to $n(p-1)-1$, where $n=\operatorname{dim} L_{-1}$.

Proof. If $c_{1}, \cdots, c_{j}$ are any elements of $L_{1}$ (or $L_{0}$ or $L_{-1}$, respectively) and $v$ is an element of $L_{-1}$ then we have by the Leibniz rule that

$$
(\operatorname{ad} v)^{p}\left[\cdots\left[c_{1}, c_{2}\right], \cdots, c_{j}\right]=\sum_{i=1}^{j}\left[\cdots\left[\cdots\left[c_{1}, c_{2}\right], \cdots,(\operatorname{ad} v)^{p} c_{i}\right], \cdots, c_{j}\right] .
$$

Now, $(\operatorname{ad} v)^{p} c_{i}$ is an element of $L_{1-p}$, (or $L_{-p}$ or $L_{-1-p}$, respectively) which is zero, since $p$ is assumed to be greater than two, and the depth of $L$ is one. Because of the commutativity of $L_{-1}$, we have that $\left(\operatorname{ad} L_{-1}\right)^{n(p-1)+1} L=0$. Consequently, by the transitivity of $L$, we have $L_{m}=0$ for $m$ greater than or equal to $-1+n(p-1)$.

Note that the height of the Jacobson-Witt algebra $W_{n}$ is $n(p-1)-1$.

Because the gradation spaces of positive degree of transitive graded Lie algebras are contained in Cartan 
prolongations, those gradation spaces will be finite dimensional whenever the local part of the transitive Lie algebra is finite dimensional. Thus, when the gradation degrees of a transitive graded Lie algebra with a finitedimensional local part are bounded, the Lie algebra is itself finite dimensional.

\section{References}

[1] Kac, V.G. (1968) Simple Irreducible Graded Lie Algebras of Finite Growth. Mathematics of the USSR-Izvestiya, 2, 1271-1312 (English), Izvestiya Akademii Nauk SSSR. Seriya Matematicheskaya, 32, 1323-1367 (Russian). 